\title{
Caracterização das alterações cutâneas provocadas pelo novo Coronavírus SARS-CoV-2: uma revisão das novas evidências
}

\author{
Characterization of dermatological manifestations caused by the new Coronavirus SARS- \\ CoV-2: a review of the new evidence
}

\author{
Caracterización de las manifestaciones dermatológicas causadas por el nuevo \\ Coronavirus SARS-CoV-2: una revisión de la nueva evidencia
}

\begin{abstract}
Wellington Manoel da Silva ${ }^{1 *}$, Maria Eduarda da Silva², Willaine Balbino de Santana Silva ${ }^{3}$, Juliana Andrade dos Santos ${ }^{1}$, Midiã Carvalho Gomes ${ }^{4}$, Jessika Luana da Silva Albuquerque ${ }^{5}$, Georgia Cybelle dos Santos Silva ${ }^{5}$, Elaine Rufino Barbosa da Silva², Manuel Santana e Silva ${ }^{2}$, Gabriella Feliciano da Silva ${ }^{3}$.
\end{abstract}

\section{RESUMO}

Objetivo: Descrever as manifestações dermatológicas decorrentes da infecção por SARS-CoV-2/COVID 19. Métodos: Trata-se de uma revisão sistemática, realizada por meio da Medical Literature Analysis and Retrieval System Online (PUBMED/MEDLINE), Literatura Latino-americana e do Caribe em Ciências da Saúde (LILACS), no sítio da Organização Pan-Americana de Saúde/Organização Mundial de Saúde (PAHO/WHO), e Cochrane Library em maio de 2020. Resultados: Sendo encontrados 28 trabalhos, foram excluídos 12 artigos, 7 por não responderam à pergunta condutora, 3 por estarem indisponíveis e 2 por estarem duplicados. A amostra foi composta por 16 artigos, todos advindos do sítio da PUBMED, publicados no período de janeiro a maio de 2020. As manifestações cutâneas observadas foram; erupção cutânea eritematosa, urticária generalizada e vesículas do tipo varicela. Considerações finais: A literatura estudada aponta para importância que esses sinais cutâneos podem ofertar nesse momento da pandemia, visto que, esses pacientes podem, inconscientemente, transmitir o SARS-CoV-2 para outras pessoas e contribuem com a disseminação da infecção por COVID-19.

Palavras-chave: Infecções por coronavírus, Manifestações cutâneas, Betacoronavirus.

\section{ABSTRACT}

Objective: To describe the dermatological manifestations resulting from SARS-CoV-2 / COVID infection - 19. Methods: This is a systematic review, carried out through the Medical Literature Analysis and Retrieval System Online (PUBMED / MEDLINE), Latin American Literature and Caribbean in Health Sciences (LILACS), on the website of the Pan American Health Organization / World Health Organization (PAHO / WHO), and Cochrane Library in May 2020. Results: Since 28 papers were found, 12 were excluded articles, 7 for not answering the conductive question, 3 for being unavailable and 2 for being duplicated. The sample consisted of 16 articles, all from the PUBMED website, published between January and May 2020. The skin manifestations observed were; erythematous rash, generalized urticaria and varicella-like vesicles. Final considerations: The studied literature points to the importance that these skin signs can offer at this time of the pandemic, since these patients can unconsciously transmit SARS-CoV-2 to other people and contribute to the spread of COVID-19 infection.

Keywords: Coronavirus infections, Cutaneous manifestations, Betacoronavirus.

\footnotetext{
${ }^{1}$ Instituto de Medicina Integral Professor Fernando Figueira (IMIP), Recife - PE.

*E-mail: wellingtonm-manoel@outlook.com

2Universidade Federal de Pernambuco (UFPE), Vitória de Santo Antão - PE.

${ }^{3}$ Universidade de Pernambuco (UPE), Recife - PE.

${ }^{4}$ Faculdade Maurício de Nassau Aliança (UNINASSAU), Teresina - PI.

${ }^{5}$ Centro Universitário da Vitória (UNIVISA), Vitória de Santo Antão - PE
} 


\section{RESUMEN}

Objetivo: Describir las manifestaciones dermatológicas resultantes de la infección por SARS-CoV-2 / COVID - 19. Metodos: Esta es una revisión sistemática, realizado a través del Sistema de análisis y recuperación de literatura médica en línea (PUBMED / MEDLINE), Literatura latinoamericana y Caribbean in Health Sciences (LILACS), en el sitio web de la Organización Panamericana de la Salud / Organización Mundial de la Salud (OPS / OMS) y la Biblioteca Cochrane en mayo de 2020. Resultados: Dado que se encontraron 28 documentos, 12 fueron excluidos artículos, 7 por no responder la pregunta conductiva, 3 por no estar disponible y 2 por estar duplicados. La muestra constaba de 16 artículos, todos del sitio web PUBMED, publicados entre enero y mayo de 2020. Las manifestaciones cutáneas observadas fueron; erupción eritematosa, urticaria generalizada y vesículas parecidas a varicela. Consideraciones finales: La literatura estudiada señala la importancia que estos signos cutáneos pueden ofrecer en este momento de la pandemia, ya que estos pacientes pueden transmitir inconscientemente el SARS-CoV-2 a otras personas y contribuir a la propagación de la infección por COVID-19.

Palabras clave: Infecciones por el coronavírus, Manifestaciones cutáneas, Betacoronavirus.

\section{INTRODUÇÃO}

Os vírus da família Coronavírus (CoVs), são classificados como agentes virais de RNA de fita simples, capazes de infectar animais e seres humanos, responsáveis por causar doenças no trato respiratório e gastrointestinais, assim como, hepáticas e neurológicas (WEISS SR, 2011). Os CoVs podem ser divididos em quatro gêneros: Alfacoronavírus, Beta-coronavírus, Gama-coronavírus e Delta-coronavírus (YANG D e LEIBOWITZ JL, 2015).

Até então haviam seis tipos de coronavírus humano (HcoVs) relatados na literatura, são estes os AlfaCoVs HCoVs-NL63, HCoVs-229E, os Beta-CoVs HCoVs-OC43, HCoVs-HKU1, o SARS-CoV, causador da síndrome respiratória aguda grave, e o MERS-CoV agente causador da síndrome respiratória do Oriente Médio (ZAKI AM, et al., 2012).

Novos Coronavírus tem emergido com frequência em humanos, causando surtos de relevância mundial, devido à alta prevalência e distribuição vasta de Coronavírus, à facilidade de recombinação genética, e ao aumento das atividades de interação entre humanos e animais (BRASIL, 2020).

No fim do ano de 2019, um novo vírus surgiu em Wuhan, província de Hubei, China, causando a chamada síndrome respiratória aguda grave, o mesmo seria posteriormente nomeado SARS-CoV-2 pelo Comitê Internacional de Taxonomia de Vírus (ICTV).

Este agente infeccioso penetra nas células por meio da enzima de conversão da angiotensina (ECA), localizada na parte apical das células. O primeiro órgão afetado pela infecção por COVID-19 são os pulmões (SACHDEVA M, et al., 2020).

Devido à sua alta infectividade e transmissão assintomática, a disseminação comunitária foi incontrolável, levando a Organização Mundial da Saúde (OMS) a declarar situação de Pandemia no mês de março, o cenário mundial já apresenta 5.998 .070 casos confirmados até o dia 30 de maio de 2020 (WANG D, et al., 2020; YANG D e LEIBOWITZ JL, 2020; WU F, et al., 2020, WHO, 2020).

O modo de transmissão acontece por meio da liberação de gotículas respiratórias ou contato com objeto contaminado com gotículas contendo o vírus (TAMARO A, 2020). Os principais sintomas observados nos acometidos de COVID-19 são tosse seca, febre, corrimento nasal, dispnéia, anorexia, náusea, diarreia e mais recentemente foram observados anosmia e disgeusia em grande quantidade de indivíduos infectados (HUI D, 2020).

Além destes, o indivíduo doente pode apresentar ainda, leucopenia, trombocitopenia e aumento dos Níveis do dímero $\mathrm{D}$, aumentando o risco de tromboembolismo venoso. Novas evidências sugerem que a liberação exacerbada de citocinas pró-inflamatórias resultam na síndrome da tempestade de citocinas, que atua facilitando o progresso de adoecimento e desenvolvimento de complicações graves. (OLIVE MM, et al., 2020). 
Em casos graves, os indivíduos podem progredir ainda para disfunção da coagulação, Síndrome Respiratória Aguda Grave (SRAG), arritmia e choque (WANG D, et al., 2020). A doença leva aproximadamente 5 dias para a manifestação dos primeiros sintomas, mas esse período pode variar de 2 a 14 dias (HUI D, et al., 2020).

O diagnóstico laboratorial é realizado por meio do exame de reação em cadeia da polimerase com transcrição reversa em tempo real (RT-PCR) de swab nasofaríngeo para identificar o material genético viral nas amostras respiratórias ou sanguíneas (YANG D e LEIBOWITZ, 2020).

A pele é considerada, pela comunidade médica, um órgão do corpo capaz de manifestar sinais de doenças internas, podendo indicar quando ocorrem disfunções metabólicas, neoplasias internas, doenças nutricionais, reações adversas a medicamentos ou doenças infecciosas sistêmicas, como as causadas pelos vírus da família Flaviviridae, Herpesviridae, Poxviridae, Papovaviridae, e Picornaviridae, entre outros causadores de doenças como, sarampo, escarlatina, doença de Chagas, tuberculose, sífilis, hanseníase, micoses profundas, leishmaniose e outras (ADIŞEN E. et al., 2015).

Sendo a pele, o órgão responsável por revelar a pista do surgimento de outra pandemia nos anos de 1980 ao mundo, o HIV/AIDS, através da produção de um estudo que alertou a ocorrência do Sarcoma de Kaposi em indivíduos com pneumonia provocada pelo então chamado Pneumocystis carinii (atualmente $P$. jirovecii), os quais estavam sendo admitidos em Los Angeles e região (California, EUA) (KIEN FAE, 1981).

Novas evidências tem apontado que o Novo Coronavírus tem provocado manifestações cutâneas diversas em pacientes de várias localizações do mundo, desta forma, este estudo se propõe a investigar, analisar, caracterizar e discutir estas evidências, afim de que, possa contribuir para o entendimento e enfrentamento da pandemia.

\section{MÉTODOS}

Este estudo trata-se de uma revisão sistemática da literatura, com o objetivo de realizar a identificação, análise e síntese dos resultados de estudos, a fim de, apresentar como se encontra a publicação científica capaz de resolver a seguinte questão norteadora: "Quais as características das lesões de pele provocadas pela infecção do SARS-CoV-2/COVID-19?".

Optou-se por este método, pois a revisão de literatura reúne e sintetiza resultados de trabalhos de maneira organizada, sobre um determinado tema contribuindo assim com o aprofundamento do conhecimento sobre o tema investigado.

Utilizou-se as etapas recomendadas a seguir: seleção de questão norteadora; definição das características das pesquisas da amostra; seleção das pesquisas que compuseram a amostra da revisão; análise dos artigos incluídos na revisão; interpretação dos resultados e discussão dos achados (LAKATOS EM e MARCONI MA, 2018).

O levantamento dos artigos foi realizado em maio de 2020, por meio do acesso on-line aos bancos da Medical Literature Analysis and Retrieval System Online (PUBMED/MEDLINE), Literatura Latino-americana e do Caribe em Ciências da Saúde (LILACS), no sítio da Organização Pan-Americana de Saúde/Organização Mundial de Saúde (PAHO/WHO), International Clinical Trials Registry Platform (ICTRP) e Cochrane Library, onde a averiguação dos artigos é feita de forma ampla.

Os descritores foram pesquisados no idioma inglês advindos do Medical Suject Headings (MeSH): "Betacoronavirus", "Coronavirus infections", "cutaneous manifestations", usando o conectivo booleano "AND" para realizar o cruzamento das palavras-chave, utilizou-se ainda, como critérios de inclusão, artigos publicados no período de janeiro de 2020 à maio de 2020, sendo estes trabalhos completos, assim como cartas ao editor, devido a relevância e atualidade do tema.

Foram excluídos, artigos que não respondessem à pergunta condutora e que não estivessem disponíveis, conforme fluxograma de representação da seleção dos artigos (Figura 1). Utilizaram-se, para a seleção das publicações incluídas no estudo, as recomendações do PRISMA. 
Figura 1 - Fluxograma do processo de seleção de trabalhos para Revisão.

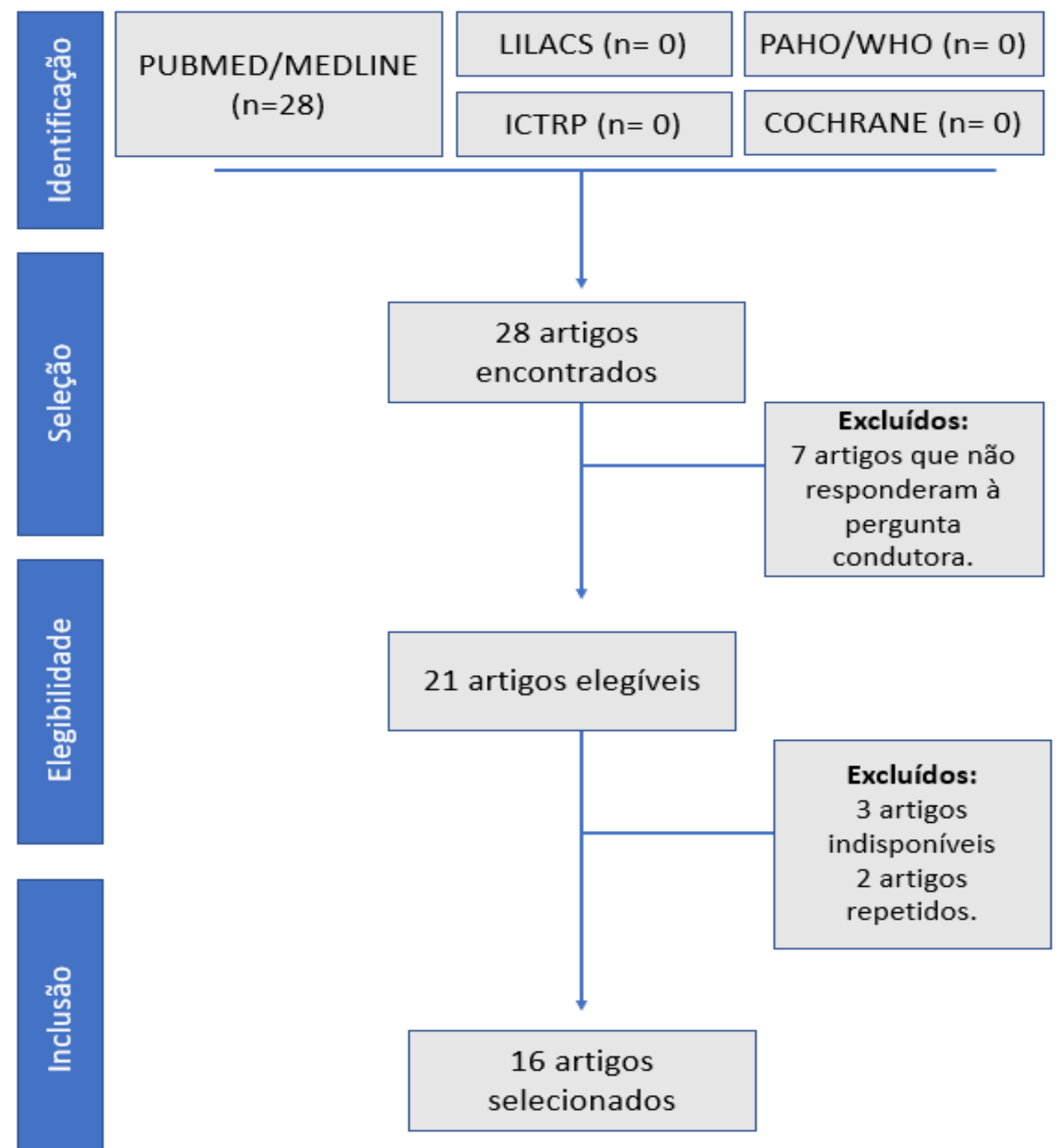

Fonte: Silva WM, et al., 2020.

\section{RESULTADOS E DISCUSSÃO}

Do total de 28 trabalhos encontrados, após leitura na íntegra, foram excluídos doze. Destes, sete por não responderem à pergunta condutora, outros três, por estarem indisponíveis, e ainda outros dois por estarem repetidos na base de dados.

Foram selecionados 16 artigos, todos os trabalhos encontrados relatam achados iniciais, tratam-se em sua maioria de cartas ao editor, relatando evidências novas sobre as alterações dermatológicas vivenciadas pelos pacientes acometidos da COVID-19.

Os estudos 2, 3, 7, 13 e 16 tratam-se de revisões de literatura, enquanto que os demais estudos são observacionais do tipo relato de caso, a saber, 1, 4, 5, 6, 8, 9, 10,11 e 12, o estudo 15 se propôs a realizar a discussão de três casos e fazer uma breve revisão para dar suporte ao conteúdo do trabalho. Os Dezesseis artigos foram organizados por título, referência e numeração de DOI (Quadro 1). 
Quadro 1 - Identificação dos estudos selecionados.

\begin{tabular}{|c|c|c|c|}
\hline $\begin{array}{l}\text { Estudo } \\
\text { (N) }\end{array}$ & Título & Autores, Ano & DOI \\
\hline 1 & $\begin{array}{c}\text { Cutaneous manifestations in COVID-19: } \\
\text { a first perspective. }\end{array}$ & RECALCATI S. 2020 & $\begin{array}{l}\text { DOI: } 10.1111 / \\
\text { JDV.16387 }\end{array}$ \\
\hline 2 & $\begin{array}{c}\text { Coronavirus Disease-2019 with } \\
\text { Dermatologic Manifestations and } \\
\text { Implications: An Unfolding Conundrum. }\end{array}$ & ALMUTAIRI N, et al., 2020 & $\begin{array}{l}\text { DOI: } 10.1111 / \\
\text { dth. } 13544\end{array}$ \\
\hline 3 & $\begin{array}{l}\text { Cutaneous Signs in COVID-19 Patients: } \\
\text { A Review. }\end{array}$ & UWE W, et al. 2020 & $\begin{array}{l}\text { DOI: } 10.1111 / \\
\text { dth.13549 }\end{array}$ \\
\hline 4 & $\begin{array}{l}\text { Erythema multiforme-like eruption in } \\
\text { patients with COVID-19 infection: clinical } \\
\text { and histological findings. }\end{array}$ & CAUHE JJ, et al. 2020 & $\begin{array}{l}\text { DOI: } 10.1111 / \\
\text { CED. } 14281\end{array}$ \\
\hline 5 & Urticarial eruption in COVID-19 infection. & HENRY D, et al. 2020 & $\begin{array}{l}\text { DOI: } 10.1111 / \\
\text { JDV.16472 }\end{array}$ \\
\hline 6 & $\begin{array}{c}\text { Cutaneous manifestations in COVID-19: } \\
\text { a new contribution. }\end{array}$ & ESTÉBANEZ A, et al. 2020 & $\begin{array}{l}\text { DOI: } 10.1111 / \\
\text { jdv.16474 }\end{array}$ \\
\hline 7 & $\begin{array}{c}\text { COVID-19 and Cutaneous } \\
\text { manifestations. }\end{array}$ & $\begin{array}{l}\text { MUNGMUNGPUNTIPANTIP } \\
\text { R e WIWANITKIT V. } 2020\end{array}$ & $\begin{array}{l}\text { DOI:10.1111/ } \\
\text { JDV.16483 }\end{array}$ \\
\hline 8 & $\begin{array}{l}\text { Acute urticaria with pyrexia as the first } \\
\text { manifestations of a COVID-19 infection. }\end{array}$ & DAMME CBMV, et al. 2020 & $\begin{array}{l}\text { DOI: } 0.1111 / \\
\text { JDV. } 16523\end{array}$ \\
\hline 9 & $\begin{array}{l}\text { Cutaneous manifestations in COVID-19: } \\
\text { the experiences of Barcelona and Rome. }\end{array}$ & TAMMARO A, et al. 2020 & $\begin{array}{l}\text { DOI:0.1111/ } \\
\text { JDV.16530 }\end{array}$ \\
\hline 10 & $\begin{array}{l}\text { Acral cutaneous lesions in the Time of } \\
\text { COVID- } 19 .\end{array}$ & RECALCATI S, et al. 2020 & $\begin{array}{l}\text { DOI:10.1111/ } \\
\text { JDV.16533 }\end{array}$ \\
\hline 11 & $\begin{array}{c}\text { Pityriasis rosea as a cutaneous } \\
\text { manifestation of COVID-19 infection. }\end{array}$ & EHSANI AH, et al. 2020 & $\begin{array}{l}\text { DOI:10.1111/ } \\
\text { JDV.16579 }\end{array}$ \\
\hline 12 & $\begin{array}{l}\text { Urticarial eruption in Coronavirus } \\
\text { Disease } 2019 \text { (COVID-19) infection: a } \\
\text { case report in Tangerang, Indonesia. }\end{array}$ & GUNAWAN C, et al. 2020 & $\begin{array}{l}\text { DOI:10.1111/ } \\
\text { JDV.16622 }\end{array}$ \\
\hline 13 & $\begin{array}{l}\text { Cutaneous manifestations in COVID-19: } \\
\text { Lessons learned from current evidence. }\end{array}$ & $\begin{array}{l}\text { SUCHONWANIT P, et al. } \\
2020\end{array}$ & $\begin{array}{c}\text { DOI: } 10.1016 / \\
\text { j.jaad } \\
.2020 .04 .094\end{array}$ \\
\hline 14 & $\begin{array}{c}\text { Cutaneous manifestations in the current } \\
\text { pandemic of coronavirus infection } \\
\text { disease (COVID 2019). }\end{array}$ & OLIVÉ MM, et al. 2020 & $\begin{array}{c}\text { doi:10.1016/ } \\
\text { j.anpede.2020.04.002 }\end{array}$ \\
\hline 15 & $\begin{array}{l}\text { Cutaneous manifestations of COVID-19: } \\
\text { Report of three cases and a review of } \\
\text { literature. }\end{array}$ & SACHDEVA M, et al. 2020 & $\begin{array}{c}\text { DOI: } 0.1016 / \\
\text { j.jdermsci.2020.04.011 }\end{array}$ \\
\hline 16 & $\begin{array}{c}\text { Cutaneous manifestations of the } \\
\text { Coronavirus Disease } 2019 \text { (COVID-19): } \\
\text { a brief review. }\end{array}$ & TANG K, et al. 2020 & $\begin{array}{l}\text { DOI: } 10.1111 / \\
\text { dth. } 13528\end{array}$ \\
\hline
\end{tabular}

Fonte: Silva WM, et al., 2020.

A maioria dos trabalhos foram publicados pelo periódico Journal of The European Academy of Dermatology and Venerealogy, nove no total (artigos 4, 5, 6, 7, 8, 9, 10,11 e 12), três no periódico Dermatology Therapy (artigos 2, 3 e 16), um na revista Clinical and Experimental Dermatology (artigo 1), um no periódico Journal of the American Academy of Dermatology (estudo 13), um na revista Anales de pediatria (artigo 14) e por fim, um no periódico Journal of Dermatogical Science (Artigo 15).

As alterações cutâneas relatadas nos trabalhos analisados foram descritas, respectivamente conforme sua fonte (Quadro 2). 


\section{Revista Eletrônica Acervo Saúde / Electronic Journal Collection Health ISSN 2178-2091}

Quadro 2 - Caracterização das alterações cutâneas relatadas na Literatura.

\begin{tabular}{|c|c|c|c|c|c|}
\hline Fonte & $\mathbf{N}$ & Manifestação Cutânea & Fonte & $\mathbf{N}$ & Manifestação Cutânea \\
\hline $\begin{array}{l}\text { JIMENEZ-CAUHE J, } \\
\text { et al. } 2020\end{array}$ & 1 & $\begin{array}{l}\text { - Pápulas eritematosas } \\
\text { - Manchas eritemato-violáceas com centro } \\
\text { escuro e uma pseudo-vesícula no meio. }\end{array}$ & $\begin{array}{l}\text { TAMMARO A, et al. } \\
\qquad 2020\end{array}$ & 9 & $\begin{array}{l}\text { - Vesículas cercadas por halos eritematosos } \\
\text { acompanhados por prurido leve. }\end{array}$ \\
\hline $\begin{array}{l}\text { ALMUTAIRI NMD, et } \\
\text { al., } 2020\end{array}$ & 2 & - Pustulose eruptiva generalizada aguda atípica. & $\begin{array}{l}\text { RECALCATI S, et al. } \\
\qquad 2020\end{array}$ & 10 & $\begin{array}{l}\text { - Erupção acral de pápulas e máculas eritemato- } \\
\text { violáceas, com possível evolução bolhosa. } \\
\text { - Lesões targetoides eritematopapulares. }\end{array}$ \\
\hline UWE W, et al. 2020 & 3 & $\begin{array}{c}\text { - Acro-isquemia } \\
\text { - Erupções semelhantes a frieira nos dedos das } \\
\text { mãos e pés. }\end{array}$ & $\begin{array}{l}\text { EHSANI AH, et al. } \\
\qquad 2020\end{array}$ & 11 & $\begin{array}{c}\text { - >Placa anular eritematosa e escamosa > } \\
\text { lesões generalizadas do tipo papular e placa > } \\
\text { lesões pruriginosas. }\end{array}$ \\
\hline RECALCATI S. 2020 & 4 & $\begin{array}{l}\text { - Erupção cutânea eritematosa, urticária } \\
\text { generalizada e vesículas do tipo varicela. }\end{array}$ & $\begin{array}{l}\text { GUNAWAN C, et al. } \\
2020\end{array}$ & 12 & - Urticária pruriginosa. \\
\hline HENRY D, et al. 2020 & 5 & $\begin{array}{l}\text { - Erupção de placas eritematosas disseminadas } \\
\text { pruriginosas. }\end{array}$ & $\begin{array}{l}\text { SUCHONWANIT P, } \\
\text { et al. } 2020\end{array}$ & 13 & $\begin{array}{c}\text { - Erupção cutânea morbiforme, erupção cutânea } \\
\text { coexistindo com trombocitopenia, máculas } \\
\text { coalescentes eritematosas a purpúricas, } \\
\text { urticária generalizada e vesículas do tipo } \\
\text { varicela. }\end{array}$ \\
\hline $\begin{array}{l}\text { ESTÉBANEZ A, et al. } \\
\qquad 2020\end{array}$ & 6 & - Pápulas eritematosas-amareladas. & $\begin{array}{l}\text { OLIVÉ MM, et al. } \\
\qquad 2020\end{array}$ & 14 & $\begin{array}{l}\text { - Erupção maculopapular eritematosa, } \\
\text { confluente e não pruriginosa. } \\
\text { - Urticária aguda pruriginosa. }\end{array}$ \\
\hline $\begin{array}{l}\text { MUNGMUNGPUNTIP } \\
\text { ANTIP R e } \\
\text { WIWANITKIT V. } 2020\end{array}$ & 7 & $\begin{array}{c}\text { - Poucos pacientes apresentaram alteração } \\
\text { dermatológica significativa. }\end{array}$ & $\begin{array}{l}\text { SACHDEVA M, et al. } \\
\qquad 2020\end{array}$ & 15 & $\begin{array}{l}\text { - Erupção cutânea maculo-papular tronco } \\
\text { semelhante a doença de Grover. } \\
\text { - Exantema maculopapular difuso (morbiforme). } \\
\text { - Erupção hemorrágica macular. } \\
\text { - Erupção papular pruriginosa. }\end{array}$ \\
\hline $\begin{array}{l}\text { DAMME CBMV, et al. } \\
\qquad 2020\end{array}$ & 8 & - Rash Cutâneo pruriginoso. & TANG K, et al. 2020 & 16 & $\begin{array}{c}\text { - Lesões cutâneas eritematosas, urticárias e } \\
\text { vesiculares semelhantes a catapora ou } \\
\text { variceliforme. } \\
\text { - Petequia e rash. } \\
\text { - Livedo reticularis. } \\
\text { - Reativação do herpes simples oral vírus tipo } 1 \\
\text { (HSV-1). }\end{array}$ \\
\hline
\end{tabular}

Fonte: Silva WM, et al., 2020. 
Dos 16 artigos selecionados para o estudo, apenas três não citam o relatório feito por Recalcati S, (2020), neste, a autora observou que $20,4 \%$ dos pacientes que participaram de um estudo observacional no Lecco Hospital, Lombardy, na Itália, apresentaram manifestações dermatológicas. As manifestações cutâneas observadas foram: erupção cutânea eritematosa, urticária generalizada e vesículas do tipo varicela. $O$ tronco era a principal região envolvida (RECALCATI S, 2020).

Os achados encontrados por Tamaro A, et al (2020), ao analisar 130 pacientes afetados pelo COVID-19 no Hospital Sant'Andrea em Roma foram semelhantes, 1,5\% deles apresentaram lesões caracterizadas por vesículas cercadas por halos eritematosos e foram acompanhados por prurido leve em seu tronco que surgiram no decorrer da internação.

Achados semelhantes foram observados em cerca de um quinto dos pacientes com COVID-19 em uma Unidade Hospitalar localizada próximo das margens do lago Como, na Itália. De um total de 88 pacientes internados visitados direta ou indiretamente por dermatologistas, 20,4\%, ou seja, 18 desenvolveram manifestações cutâneas. Oito dos 18 (44\%) apresentaram erupções cutâneas no início dos sintomas, os demais após hospitalização.

Quatorze pacientes (78\%) desenvolveram erupção cutânea eritematosa, três, urticária generalizada e um apresentou vesículas do tipo catapora. A principal área afetada foi o tronco em todos os pacientes. Houve ainda prurido leve ou ausente. (ALMUTAIRI N, et al., 2020). No estudo 4, Cauhe JJ, et al. (2020), relataram por meio de um estudo observacional com 4 pacientes hospitalizados um novo padrão de lesões com eritema até então não relatado em outros estudos. Todos os pacientes, apresentaram lesões cutâneas que começaram como pápulas eritematosas na região do tronco superior, e progressivamente se voltaram para manchas eritemato-violáceas com centro escuro e uma pseudo-vesícula no meio.

Para os autores tais lesões são sugestivas e típicas das infecções causadas por vírus membros da família Herpes viridae (TAMARO A, 2020). O estudo 3 relatou alguns achados importantes por meio de revisões de literatura, o primeiro foi a descrição de casos em que ocorreu hipercoagulação intravascular disseminada com achados laboratoriais, como exacerbação dos níveis de dímero $D$ e degradação do fibrinogênio, além de tempo prolongado de protrombina.

Esses pacientes, acometidos de casos graves da COVID-19 apresentam Acro-isquemia com cianose dos dedos dos pés, bolhas cutâneas e gangrena seca. O segundo tratou-se de erupções edematosas e eritematosas do tipo frieira nos pés, observadas em casos mais leves de COVID-19, que desaparecem após a infecção e não fizeram cicatrizes.

Por vezes associados a coceira ou dor e com duração média de 2 semanas. Os autores reportam ainda que estas lesões foram observadas em 25 pacientes, com idade média de 14 anos, na Espanha. Destes, nenhum apresentou outros sintomas típicos do COVID-19, exceto um, que apresentou diarreia (MORENO RA, 2020). Uwe W, et al., (2020) observaram ainda o eritema multiforme como erupção cutânea ocorrendo em 27 crianças com sintomatologia leve, duas desenvolveram lesões targetoides. Nestes, foi descartado 0 diagnóstico de infecção por Herpes simplex, diferentemente do que sugeriam trabalhos anteriores como o estudo 4.

No qual, os autores apontaram que o surgimento de eritema multiforme está ligado a infecção por agentes infecciosos em $90 \%$ dos casos, enquanto que, surge como reação alérgica à medicamentos em menos de $10 \%$ dos casos. O vírus do Herpes simplex e o Mycoplasma pneumoniae foram relatados no estudo como os principais responsáveis pelo surgimento do eritema multiforme (CASAS CG, 2020).

No estudo 5, Henry D, et al., (2020) relataram o caso de uma Residente Médica que apresentou, inicialmente, erupção de placas eritematosas disseminadas pruriginosas, recebendo o diagnóstico de urticária. Em cerca de 48 horas evoluiu com febre e dor torácica e testou positivo para COVID-19. O caso chamou a atenção dos autores por sugerir o desenvolvimento de manifestações cutâneas antes mesmo do aparecimento de sintomas como febre e dificuldade respiratória. Este achado condiz com outros estudos analisados, a saber, estudos 5, 6, 8 e 16, onde os autores relatam que erupções cutâneas do tipo urticariforme com petéquias tem sido descritas como sintomas e/ou sinais iniciais da COVID-19. O estudo 6 , ainda cita 0 
acometimento de edema hemorrágico agudo em pacientes pediátricos associado ao coronavírus (ESTÉBANEZ A, et al. 2020; DAMME CBMV, et al. 2020; HENRY D, et al. 2020; TANG K, et al., 2020). Entretanto, outro estudo (estudo 10) relatou casos nos quais observou-se o aparecimento de alterações cutâneas precedidas dos sintomas de febre e dificuldade respiratória (RECALCATI S, et al., 2020).

Para Estébanez A, et al (2020) é difícil identificar se as manifestações decorrem de infecção viral ou alergia à nova medicação prescrita. Tal afirmativa é defendida também por Olivé MM, et al (2020), autores do estudo 14 , contudo outros trabalhos conseguiram verificar o desenvolvimento de alterações dermatológicas em pacientes infectados por SARS-CoV-2, descartando a hipótese de reação alérgica medicamentosa.

No estudo 10, Recalcati S, et al., (2020) observaram ao analisar 14 casos de indivíduos jovens em uma unidade dermatológica, que haviam desenvolvido manifestações cutâneas, do tipo erupção acral de pápulas e máculas eritemato-violáceas, com evolução bolhosa ou edema digital, que em nenhum dos casos houve associação com exposição ao frio, comorbidades ou ingestão de drogas e/ou medicamentos.

O mesmo ocorre no estudo 11, no qual, os autores relataram um caso de pitiríase rósea em um paciente com histórico familiar e de contato recente, confirmado para COVID-19, que teria apresentado inicialmente febre baixa, fadiga, gastroenterite e anorexia. Evoluindo, posteriormente, para uma placa anular eritematosa e escamosa, dias depois, lesões generalizadas do tipo papular.

As lesões continuaram se disseminando por uma semana e se tornaram pruriginosas. Segundo os autores, o paciente apresentava longa história de ingestão de sertralina e propranolol e não houve nenhuma alteração no regime de tratamento nos últimos meses, descartando-se reações alérgicas. Este paciente acabou não recebendo confirmação da doença viral e foi tratado ambulatorialmente (EHSANI AH, et al. 2020).

Gunawan C, et al. (2020) relataram o caso de um paciente positivo para COVID-19 com urticária em Unidade de Terapia Intensiva, na Indonésia. Paciente idoso, internado em 12 de abril de 2020 apresentou os sintomas de febre, tosse, dispnéia e diarréia. Teve confirmação da doença por meio do resultado positivo do RT-PCR para COVID-19. O mesmo apresenta ainda as seguintes comorbidades: hipertensão, diabetes, dislipidemia e hiperuricemia em terapia. O paciente recebeu durante sua hospitalização tratamento medicamentosos com azitromicina, hidroxicloroquina, cefoperazona-sulbactam, omeprazol e medicamentos para suas comorbidades.

Em pouco tempo de hospitalização, foi observado o surgimento de urticária pruriginosa envolvendo a face e sem envolvimento do resto da pele. Apenas 5 dias após o início dos sintomas. Todavia, não foram encontrados outros possíveis desencadeadores de urticária, além da infecção pelo SARS-CoV-2, pois não haviam registros no seu histórico acerca de alergias a medicamentos, urticária crônica, ou outras alergias. Os autores enfatizam que não havia histórico de consumo de nenhum novo medicamento no espaço de tempo de 15 dias antes, além daqueles utilizados para tratamento do COVID-19 no hospital.

No estudo 15, Sachdeva M, et al. (2020) relataram três casos, o primeiro tratou-se de uma idosa de 71 anos de idade, que se apresentou à Emergência Departamento de Milão, queixando-se de febre, tosse produtiva e falta de ar, apresentando teste laboratorial positivo para a doença COVID-19. Até o momento a paciente era saudável, sem comorbidades, sem uso de medicamentos nem relatos de reações adversas medicamentosas anteriores. Após dias de internamento, ocorreu o surgimento de uma erupção cutânea maculo-papular no tronco semelhante a doença de Grover.

Para Almutairi N, et al, (2020), essas manifestações dermatológicas surgem em decorrência de interações medicamentosas ou reações adversas induzidas por medicamentos experimentais utilizados no tratamento dos infectados por COVID-19, visto que, até o presente momento, não existem tratamentos com comprovada eficácia para tratamento dos doentes.

Segundo os autores, os medicamentos cloroquina e hidroxicloroquina que estão sendo utilizados de forma empírica podem agravar um quadro de psoríase preexistente ou produzir uma variedade de reações cutâneas. Os autores destacam, ainda, o eritema figurado pustular generalizado, reação dermatológica grave potencialmente fatal, recentemente classificada como pustulose eruptiva generalizada aguda atípica. $O$ 
segundo caso relatado nesse estudo, tratou-se de uma mulher de 77 anos, tendo obtido teste de RT-PCR positivo para COVID-19, foi internada em um hospital de Milão.

A mesma apresentou febre, tosse e exantema maculopapular difuso (morbiforme) no tronco. Pouco tempo depois, ela também desenvolveu erupção hemorrágica macular nas pernas. O tratamento implementado consistiu no uso do lopinavir e hidroxicloroquina, as lesões apresentaram melhora espontânea em poucos dias. O terceiro caso relatado pelo estudo 15 foi de uma idosa, 72 anos, sem comorbidades, que procurou 0 Departamento de Emergência em Milão com dor de cabeça, artralgia, mialgia e febre.

Uma semana depois, desenvolveu um quadro de erupção vesicular pruriginosa nas regiões sub-mamária, tronco e quadris. Foi realizado teste com swab nasofaríngeo para COVID-19, e o resultado positivo. Remissão completa de todos os sintomas, gerais e das manifestações cutâneas foram observadas em aproximadamente dez dias (SACHDEVA M, et al. 2020). Na maioria dos casos relatados nos estudos que foram analisados, o período entre o surgimento e remissão das alterações cutâneas são de 5 a 10 dias. Nos casos em que as manifestações dermatológicas antecederam os demais sintomas, a duração foi de 5 dias.

Os estudos apresentados, caracterizaram as principais manifestações dermatológicas apresentadas pelos indivíduos acometidos por COVID-19 sendo estas: Pápulas eritematosas (em cinco estudos), manchas eritemato-violáceas com centro escuro e uma pseudo-vesícula no meio (em um estudo), pustulose eruptiva generalizada aguda atípica (em um estudo), acro-isquemia (em dois estudos), erupções semelhantes a frieira nos dedos das mãos e pés (em um estudo), urticária generalizada e vesículas do tipo varicela (em quatro estudos), erupção de placas eritematosas disseminadas pruriginosas (em três estudos), rash cutâneo pruriginoso (em dois estudos), lesões targetoides eritematopapulares (em um estudo), erupção cutânea morbiforme (em um estudo), erupção cutânea maculo-papular tronco semelhante a doença de Grover (em um estudo), erupção hemorrágica macular (em um estudo) e Livedo reticularis (em um estudo) (Quadro 2).

\section{CONSIDERAÇÕES FINAIS}

Os achados contidos nesta revisão basearam-se nas experiências iniciais com a temática abordada, devido a limitação referente a disponibilidade da literatura sobre as alterações dermatológicas e COVID-19, visto que, este artigo examina apenas 139 casos de manifestações cutâneas relacionadas ao COVID-19 relatadas no mundo a partir de janeiro de 2020. Contudo, por meio da coleta das evidências mais recentes, sugere-se que a COVID-19 pode ter manifestações cutâneas. Contudo, fazem necessários novos estudos, a fim de fortalecer as evidências científicas e o processo de combate à pandemia e proporcionar uma melhor compreensão do assunto. A literatura estudada aponta para importância que esses sinais cutâneos podem ofertar nesse momento da pandemia, visto que, esses pacientes podem, inconscientemente, transmitir o SARS-CoV-2 para outras pessoas e contribuem com a disseminação da infecção por COVID-19, destarte, ressalta-se a necessidade de isolamento. Torna-se essencial levar em consideração e promover o potencial clínico entre as desordens cutâneas e a infecção por covid-19, proporcionando a testagem para o COVID-19 nesses casos.

\section{REFERÊNCIAS}

1. ADIŞEN E, ÖNDER M. Viral infections of the folds (intertriginous areas). Clinics in Dermatology, 2015;33(4):429-436.

2. AWULACHEW MT. Coronavirus Disease (COVID-19) Outbreak: A Review Article. International Journal of Virology and Diseases, 2020.

3. CASAS GC, et al. Classification of the cutaneous manifestations of COVID-19: a rapid prospective nationwide consensus study in Spain with 375 cases [published online ahead of print, 2020 Apr 29]. British Journal of Dermatology, 2020.

4. DAMME CBMV, et al. Acute urticaria with pyrexia as the first manifestations of a COVID-19 infection [published online ahead of print, 2020 Apr 24]. Journal of the European Academy of Dermatology and Venereology, 2020.

5. EHSANI AH, et al. Pityriasis rosea as a cutaneous manifestation of COVID-19 infection [published online ahead of print, 2020 May 2]. Journal of the European Academy of Dermatology and Venereology, 2020; DOI: 10.1111/jdv.16579.

6. ESTÉBANEZ A, et al. Cutaneous manifestations in COVID-19: a new contribution [published online ahead of print, 2020 Apr 15]. Journal of the European Academy of Dermatology and Venereology, 2020; DOI: 10.1111/jdv.16474. 
7. FERNANDEZ-NIETO D, et al. Commenton: Cutaneous manifestations in COVID-19: a first perspective. Safety concerns of clinical images and skin biopsies. Journal of the European Academy of Dermatology and Venereology, 2020.

8. HENRY D, et al. Urticarial eruption in COVID-19 infection [published online ahead of print, 2020 Apr 15]. Journal of the European Academy of Dermatology and Venereology, 2020; DOI: 10.1111/jdv.16472.

9. HUI DSI, et al. The continuing 2019-nCoV epidemic threat of novel coronaviruses to global health - Thelatest 2019 novel coronavirus outbreak in Wuhan, China. International Journal Infectious Diseases, 2020; DOI: 91:254-266.

10. KIEN FAE. Disseminated Kaposi's sarcoma syndrome in young homosexual men. Journal of the American Academy of Dermatology, 1981; 5: 468-71.

11. MARCONI MA, LAKATOS EM. Técnicas de pesquisa. 6. ed. São Paulo: Editora Atlas, 2018.

12. MORENO AR, et al. Cutaneous manifestation of COVID-19 in images: A case report [published online ahead of print, 2020 Apr 24]. Journal of the European Academy of Dermatology and Venereology, 2020; DOI: 10.1111/jdv.16531.

13. MOREY-OLIVÉ M, et al. Cutaneous manifestations in the current pandemic of coronavirus infection disease (covid 2019). Anales de Pediatria, 2020; DOI: 10.1016/j.anpede.2020.04.002.

14. MOREY-OLIVE M, et al. Manifestacion escutánea sen contexto del brote actual de enfermedad por coronavirus 2019. Anales de Pediatria, 2020.

15. MUNGMUNGPUNTIPANTIP R, WIWANITKIT V. COVID-19 and Cutaneous manifestations [published online ahead of print, 2020 Apr 15]. Journal of the European Academy of Dermatology and Venereology, 2020.

16. RECALCATI S, et al. Acral cutaneous lesions in the Time of COVID-19 [published online ahead of print, 2020 Apr 24]. Journal of the European Academy of Dermatology and Venereology, 2020.

17. RECALCATI S. Cutaneous manifestations in COVID-19: a first perspective [published online ahead of print, $2020 \mathrm{Mar}$ 26]. Journal of the European Academy of Dermatology and Venereology, 2020.

18. REINHARD A, et al. Anosmie et COVID-19 [Anosmia and COVID-19]. Revue Médicale Suisse, 2020; 16(691-2): 849851.

19. SACHDEVA M, et al. Cutaneous manifestations of COVID-19: Report of three cases and a review of literature. Journal of Dermatological Science, 2020.

20. SUCHONWANIT P, et al. Cutaneous manifestations in COVID-19: Lessons learned from current evidence. Journal of the American Academy of Dermatology, 2020.

21. TAMMARO A, et al. Cutaneous manifestations in COVID-19: the experiences of Barcelona and Rome [published online ahead of print, 2020 Apr 24]. Journal of the European Academy of Dermatology and Venereology, 2020.

22. WANG D, et al. Clinical Characteristics of 138 Hospitalized Patients With 2019 Novel Coronavirus-Infected Pneumonia in Wuhan, China. Journal of the American Medical Association, 2020.

23. WEISS SR, LEIBOWITZ JL. Coronavirus pathogenesis. Advances in Virus Research, 2020.

24. WU F, et al. A new coronavirus associated with human respiratory disease in China. Nature, 2020.

25. YANG D, LEIBOWITZ JL. The structure and functions of coronavirus genomic 3' and 5' ends. Virus Research, 2015.

26. YANG P, WANG X. COVID-19: a new challenge for human beings. Cellular\& Molecular Immunology, 2020; 1-3.

27. ZAKI AM, et al. Isolation of a novel coronavirus from a man with pneumonia in Saudi Arabia. New England Journal of Medicine, 2012. 